\title{
New contributions to the flora of León
}

\section{Linda González de Paz (*), Sara del Río (**) \& Ángel Penas (**)}

\begin{abstract}
González de Paz, L., del Río, S. \& Penas, A. New contributions to the flora of León. Lazaroa 35: 209-219 (2014).

Chorological and taxonomical data on several taxa from the province of León are reported . For some we provide the first record for the province, or extend their known distribution.
\end{abstract}

Keywords: Checklist, León, Spain, Chorology, Taxonomy. (2014).

Resumen: González de Paz, L., del Río, S. \& Penas, A. Nuevas aportaciones a la flora de León. Lazaroa 35: 209-219

Aportamos datos sobre la distribución y taxonomía de distintos táxones en la provincia de León, entre los que destacan primeras referencias provinciales y ampliación de su distribución conocida.

Palabras clave: Flora, León, España, Corología, Taxonomía.

\section{INTRODUCTION}

This paper contains the first reports of nine taxa for the province of León, and the extension of the geographic distribution of these species in the region. New records of range extensions are recorded for some plant taxa.

The voucher specimens collected by us and reported here include the collection location -with its municipality in parentheses-, UTM coordinates, elevation, habitat, collection date, number assigned in the University of León Herbarium collection (LEB) and the collectors name. For the voucher specimens not collected by us, we include the information provided on their label.

Taxa names follow Flora Iberica (CASTROVIEJO \& al., 1986-2013) and Flora Europaea (TuTin \& al., 1968-1980, 1993), except in some cases where we have followed the criteria of taxonomic specialists: Phelipanche (CARLÓn \& al., 2005), Tolpis (TAlaVERA, 1980) and Klasea (CANTÓ, $1984,2012,2013)$. Other reference works for the area are: Del Egido \& Puente (2011), Del EgIDO \& al. (2007, 2012b).

\section{RESULTS AND DICUSSION}

\section{Aira cupaniana Guss.}

León: Between Puente de Domingo Flórez and Vega de Yeres (Puente de Domingo Flórez), 29TPG7998, 430 m, grasslands, SE, 14.04.2003, L. González de Paz, LEB 99389. Balouta River valley in Salas de la Ribera (Puente de Domingo Flórez), 29TPH8001, 470 m, grasslands, NW, 29.04.2004, L. González de Paz, LEB 99388. Between La Baña and Silván (Encinedo), 29TPG9285, $1500 \mathrm{~m}$, grasslands, S-SW, 12.06.2004, L. González de Paz, LEB 97780.

Although this species has been reported for the nearby province of Orense (LAínZ, 1974: 23; GIMÉNEZ DE AZCÁRATE \& al., 1990: 11; GIMÉNEZ DE

\footnotetext{
* Biodiversity and Environmental Management Department (Botany). University of León. Campus de Vegazana, s/n. E-24071. León.

** Mountain Livestock Farming Institute (Joint Center CSIC-ULE). Biodiversity and Environmental Management Department (Botany). University of León. Campus de Vegazana, s/n. 24071. León.
} 
AZCÁRATE \& AMIGO, 1996: 21), we have not found bibliographic references of it or herbarium vouchers collected in our province. Herein, we report the first voucher specimen data confirming its occurrence in León.

\section{Brachypodium retusum P.Beauv.}

(= Brachypodium ramosum Roem. \& Schult.)

León: Near the peak Cruz de la Peña (Benuza), 29TPH9003, $1342 \mathrm{~m}$, base-rich grasslands, SE, 11.07.2007, L. González de Paz, LEB 99261.

It has been reported from Los Barrios de Luna (Llamas \& ACEDO, 1997: 141) and Corsalines valley (LENCE PAZ, 2001: 86), a third additional record for León is provided increasing the range of this species to the southwest of León.

Carex depauperata Curtis ex With.

León: Between Benuza and Yebra (Benuza), 29TPG896981, 700 m, oak forest, NE, 17.05.2005, L. González de Paz, LEB 96895. Santa Elena chapel pathways (Benuza), 29TPG9392, $982 \mathrm{~m}$, oak forest, NW, 11.06.2006, L. González de Paz, LEB 96894.

Reports of Carex depauperata in León are documented in Vicioso (1959: 154) but without a precise locality. Molina \& al. (2006: 31) provided records from El Bierzo (Molinaseca, Toreno and Bouzas).

\section{Carex furva Webb}

León: Cadabal River glacial cirque (Encinedo), 29TPG8777, $2022 \mathrm{~m}$, chionophilous matgrass communities, bogs and alpine grasslands, 14.08.2006, L. González de Paz, LEB 96628.

LUCEÑO (1986: 437) provides the only known report from León, collected in Peña Trevinca. Our citation adds a second record.

Carex pendula Huds.

León: Cabrera River in Vega de Yeres (Puente de Domingo Flórez), 29TPG8298, 400 m, riverbanks,
11.06.2005, L. González de Paz, LEB 96819. Pombriego (Benuza), 29TPG896989, $480 \mathrm{~m}$, stream border, 30.04.2006, L. González de Paz, LEB 96820.

We have found citations from the Luna River valley (RoMERo RodríGUEZ, 1983: 139), Vegabaño (CARbó \& al., 1987: 73) and La Pola de Gordón (PÉrez Morales, 1988: 203). We provide additional localities for this sedge.

\section{Carex sylvatica Huds. subsp. sylvatica}

León: Cabrera River in Llamas de Cabrera (Benuza), 29TPG9497, $560 \mathrm{~m}$, hazel forest, NW, 11.08.2009, L. González de Paz, LEB 103041.

Although this species is widely distributed in the north of León, it has not been previously reported in the southwest. This record extends the range of this taxon in southwestern territories of León.

\section{Centaurium maritimum (L.) Fritsch}

León: Limestones in Ferradillo (Benuza), 29TPH9003, $1389 \mathrm{~m}$, base-rich grasslands, S, 11.07.2007, L. González de Paz, LEB 94753.

Pérez CARro \& al. (1985: 139) recorded it from Villadangos del Páramo and AEDO \& al. (2001: 26) from Cabañas Raras. This is the third record for León. It has been also cited in Orense (LAÍnZ, 1971: 18; MorlA, 1982: 394) and Zamora (GARCía Río \& NAVARRo ANDRÉs, 1994: $56)$.

\section{Cheilanthes acrostica (Balb.) Tod.}

León: Peñarrubia, Portela del Cuerno (Carucedo), 29TPH8003, $860 \mathrm{~m}$, limestone fisures, S, 15.04.2003, L. González de Paz, LEB 95362. Salas de la Ribera (Puente de Domingo Flórez), 29TPH8102, $800 \mathrm{~m}$, limestone fisures, S, 05.04.2004, L. González de Paz, LEB 95361. Salas de la Ribera, Vanicelos stream valley (Puente de Domingo Flórez), 29TPH8002, 692 m, limestone fisures, S, 16.05.2007, L. González de Paz, LEB 95360. Salas de la Ribera, Balouta stream valley (Puente de Domingo Flórez), 
29TPH8002, $690 \mathrm{~m}$, limestone fisures, SW, 10.08.2009, L. González de Paz, LEB 103059. Near the Peñarrubia reservoir, 17.05.2007, T.E. Díaz \& al., LEB 15204.

Orense: Peñarrubia, S, 23.05.1982, F. Llamas \& J. Andrés, LEB 11200. Rubiana, 29TPH70, dolomite fisures, S, 11.04.1983, F.J. Pérez Carro \& M.P. Fernández Areces, LEB 31324. Rubiana, dolomite fisures, S, 11 .06.1983, F.J. Pérez Carro \& M.P. Fernández Areces, LEB 23279. Rubiana, dolomite fisures, S, 11 .06.1983, F.J. Pérez Carro \& M.P. Fernández Areces, LEB 29231.

This taxon has only been cited from León by LANGE (1860: 22 sub Cheilanthes odora Sw.) although AEDO \& al. (2001:9) thought it should be Cheilantes tinaei, because is abundant in the area. We include under this species some specimes determined as Cheilanthes pteridioides (Reichard) C.Chr. after revising LEB. Cheilanthes acrostica has not without glandular hairs under the pinnae and the pseudoindusium is frimbriate.

\section{Datura innoxia Mill.}

León: Benavides de Órbigo (Benavides de Órbigo), 30TTN617099, $845 \mathrm{~m}$, disturbed soils, 17.09.2013, L. González de Paz, LEB 107348.

This Mexican and Central American native has not been previously reported in León, being our record the first for the province. Different works on allochthonous Iberian or Castilla y León flora do not include León within the range of this taxon (SANZ ElORZA \& al., 2004: 150-151; ACEDO \& LlamAS, 2006; SANZ ElORZA \& al., 2008: 126) but it has been cited for Salamanca, Zamora and Valladolid (SANZ ElorZA \& GonZÁlez BERNARDO, 2007: 108).

Digitaria ischaemum (Schreb.) Muhl.

León: Castroquilame (Puente de Domingo Flórez), 29TPG8599, 422 m, abandoned gardens, 11.08.2009, L. González de Paz, LEB 103044.

Herein, we document the second record of this invasive weed for León. It has previously been documented in our province from Valderrey (LAínZ, 1973: 204).

\section{Eriophorum angustifolium Honck.}

León: La Baña Lake (Encinedo), 29TPG8580, 1420 m, bogs, 21.07.2010, L. González de Paz, LEB 105330. Peña Trevinca, 29TPG88, 16.07.1982, $F$. Llamas, LEB 50485.

Although this species is relatively commonly distributed in northern León, bibliographic references from the southwestern territories are rare. We have found a specimen in LEB from Peña Trevinca.

Euphorbia exigua L. subsp. merinoi M. Laínz

León: Portela del Cuerno, near Peñarrubia (Puente de Domingo Flórez), 29TPH8003, 860 m, dolomite fisure, S, 15.04.2003, L. González de $\mathrm{Paz}$, LEB 96689. Salas de la Ribera (Puente de Domingo Flórez), 29TPH808027, 750 m, baserich therophitic grasslands, S, 05.05.2005, L. González de Paz, LEB 96688. Portela del Cuerno, near Peñarrubia (Carucedo), 29TPH811036, 860 m, Ononido pusillae-Thymetum zygidis, S-SW, 18.05.2005, L. González de Paz, LEB 96687. Gordoncillo, 30TUM06, Tuberarietea, 21.09.1985, M.E. García, LEB 49772. San Juan de Paluezas, 29TPH80, 11.05.1986, M.E. García, LEB 33031. San Juan de Paluezas, 29TPH80, 11.05.1986, M.E. García, LEB 33031, 48850.

Alonso Redondo (2003: 142) reported this taxon near the Duerna Valley. The revision of specimens in LEB confirmed new records of subsp. merinoi collected in San Juan de Paluezas and Gordoncillo.

\section{Fumaria bastardii Boreau}

León: Sotillo de Cabrera (Benuza), 29TPG8595, 1250 m, pathway edge, SE, 11.07.2002, L. González de Paz, LEB 97858. Between Losadilla and La Baña (Encinedo), 29TPG9381, $1020 \mathrm{~m}$, road side, S, 24.06.2004, L. González de Paz, LEB 97857. Benuza (Benuza), 29TPG8896, $800 \mathrm{~m}$, gardens, 30.08.2005, L. González de Paz, LEB 97856. 
It was formerly reported by GANDOGER from Villafranca del Bierzo (1910: 95; 1917: 11). We have not found specimens at LEB just those of this paper that represent the second time this species is recorded in León.

\section{Heracleum sphondylium L. subsp. sphondylium}

León: Marrubio, near the Cabrera River (Castrillo de Cabrera), 29TQG0389, $800 \mathrm{~m}$, meadow, NE, 02.07.2002, L. González de Paz, LEB 95095. Silván, Silván River (Benuza), 29TPG9188, 1000 $\mathrm{m}$, riverbank, 22.07.2003, L. González de Paz, LEB 95094. Pequeño River near Manzaneda (Truchas), 29TQG2182, $1020 \mathrm{~m}$, riparian shrubs, 27.06.2001, L. González de Paz, LEB 95096. Marrubio, 29TQG0389, $750 \mathrm{~m}, 15.07 .1993, M$. de Godos, LEB 60737.

We have found two records from León in bibliographic references: Oseja de Sajambre (ARENAS POSADA \& GARCÍA MARTín, 1993: 187) and Villafranca del Bierzo (SILVA PANDO, 1994: 339). Our voucher specimens and another one from Marrubio, housed at the Herbarium LEB, represent the third and next reports of this taxon from León.

\section{Hieracium arevacorum Mateo}

León: La Guiana (Benuza), 29TPH9701, 1750 m, quartzite fisures, NE, 26.07.2004, L. González de Paz, LEB 100043.

We know the records by MATEO SANZ (2006: 40), and Mateo SANZ \& Del Egido (2011: 30) from Senra (Murias de Paredes).

Klasea legionensis (Lacaita) Holub (= Serratula legionensis Lacaita)

León: Sotillo de Cabrera, near Peña Nanda (Benuza), 29TPG8495, $1400 \mathrm{~m}$, heathlands, NW, 11.07.2002, L. González de Paz, LEB 96354.

We contribute the first record from León of this bercian-sanabriensean endemic taxon. It has been reported from Zamora (Sierra de Porto-Sanabria) and Orense (Serra do Xurés, Trevinca) by
CANTó (1984: 33, 2012: 23), and also occurs in Portugal near Orense (BAÑARES \& al., 2009: 74).

\section{Melilotus spicatus (Sm.) Breistr.}

León: Salas de la Ribera, Peñarrubia reservoir (Puente de Domingo Flórez), 29TPH7902, 400 m, grasslands, W-NW, 25.06.2002, L. González de Paz, LEB 96483. Sil River in Salas de la Ribera (Puente de Domingo Flórez), 29TPH7900, 380 m, stony soil, 03.06.2006, L. González de Paz, LEB 96484.

We have found two bibliographic references from León: Santa Lucía (PÉREz CARRO \& al., 1985: 138, sub M. neapolitana) and near Santa Olaja de la Varga (Alonso REDNDO \& al., 1999: 193, sub M. neapolitana Ten.), both in the Cantabrian Range.

\section{Phelipanche nana (Noë) Soják}

(= Orobanche nana (Reut.) Beck; = Orobanche ramosa L. subsp. nana (Reut.) Cout.)

León: Castrillo de Cabrera, Bárcena stream (Castrillo de Cabrera), 29TQG0191, 950 m, meadow, SE, 21.06.2002, L. González de Paz, LEB 97878. Portela del Cuerno, near Peñarrubia (Carucedo), 29TPH8003, $860 \mathrm{~m}$, crack in limestone, S, 15.04.2003, L. González de Paz, LEB 97875. Salas de la Ribera, Peñarrubia reservoir (Puente de Domingo Flórez), 29TPH7902, 450 m, baserich grasslands, W, 20.05.2003, L. González de Paz, LEB 97876. Salas de la Ribera (Puente de Domingo Flórez), 29TPH8002, 750 m, base-rich grasslands, SW, 29.04.2004, L. González de Paz, LEB 97874. Salas de la Ribera (Puente de Domingo Flórez), 29TPH808027, 780 m, Ononido pusillae-Thymetum zygidis, S, 05.05.2005, L. González de Paz, LEB 97877. Portela del Cuerno, near Peñarrubia (Carucedo), 29TPH811037, 870 $\mathrm{m}$, base-rich shrublands, S-SW, 18.05.2005, L. González de Paz, LEB 97879. Peñarrubia reservoir (Puente de Domingo Flórez), 29TPH7903, $501 \mathrm{~m}$, Ononido pusillae-Thymetum zygidis, W, 19.05.2007, L. González de Paz, LEB 97873.

We provide new citations for León of this poorly collected taxon that has been reported from 
Puente de Domingo Flórez and Peñarrubia reservoir (AEDO \& al., 2002: 29).

Pisum sativum L. subsp. elatius (M. Bieb.) Asch. \& Graebn. var. elatius

León: Between Sigüeya and Llamas (Benuza), 29TPG9495, $800 \mathrm{~m}$, pathway ditch, N, 25.05.2005, L. González de Paz, LEB 96455.

AEDO \& al. (2000: 49) and CANTORAL GONZÁLEZ \& al. (2011: 17) have also reported it. GIMÉNEZ DE AzCÁRATE \& AMIGO (1996: 112) cited it in Orense.

\section{Plumbago europaea L.}

León: Peñarrubia reservoir (Puente de Domingo Flórez), 29TPH7902, 409 m, dolomites fisures, NW, 04.08.2006, L. González de Paz, LEB 97680. Salas de la Ribera, Balouta stream valley (Puente de Domingo Flórez), 29TPH8102, 617 $\mathrm{m}$, base-rich grasslands, 10.08.2009, L. González de Paz, LEB 103055.

Although it has been recorded from Orense the limestones in Peñarrubia (LAínZ, 1968: 31) and Vilardesilva (GIMÉNEZ DE AZCÁRATE \& Amigo, 1996: 113)-, in León it was only known from Carucedo (AEDo \& al., 1994: 84). The data reported herein document the second and third records from the province.

\section{Potamogeton coloratus Hornem.}

(= Potamogeton fluitans Roth p.p.)

León: Eria River between Quintanilla de Yuso and Villar del Monte (Truchas), 29TQG1879, 1050 m, backwater, 28.06.2004, L. González de Paz, LEB 97639.

This species has been reported by ROMERo RoDRÍGUEZ (1983: 126) from the Babia Lake. We contribute with the second record.

\section{Pseudorchis albida (L.) Á. Löve \& D. Löve}

León: Castrillo de Cabrera, La Sierra stream valley (Castrillo de Cabrera), 29TPG9997, 1686 m, megaforbic community, W, 25.07.2007, L. González de Paz, LEB 97894. Peña Cuiña, 29TPH74, 1850 m, 29.07.1983, T.E.Díaz \& al., LEB 22109.

Fourth and fifth records of the known species distribution in León. It has already been cited in Puerto Dobres, in Picos de Europa (LAínz, 1976: 43 sub Leucorchis albidus (L.) E.Mey.), Candín (Silva Pando, 1994: 282) and Tejedo del Sil (AEDo, 2003).

This taxon is included in the Castilla y León Protected Flora Catalog (Decreto 63.2007, 14th june), classified as "Preferential Attention".

\section{Pyrola minor L.}

León: Faeda stream valley (Encinedo), 29TPG9279, $1204 \mathrm{~m}$, birch forest, NW, 25.08.2006, L. González de Paz, LEB 97619. Caprada River valley (Castrillo de Cabrera), 29TQG0396, 1507 m, birch forest, NE, 29.08.2006, L. González de Paz, LEB 97618.

Well documented from northern León, in the south of the province we only knew one station, near La Baña Lake (LlamAs \& al., 1984: 284). Our voucher data report additional localities for this taxon in southwestern León.

\section{Reseda virgata Boiss. \& Reut.}

León: Iruela (Truchas), 29TQG0582, $1250 \mathrm{~m}$, roadside, SW, 12.07.2001, L. González de Paz, LEB 98638. Corporales, telephone antennas (Truchas), 29TQG0885, $1500 \mathrm{~m}$, grasslands, 07.08.2001, L. González de Paz, LEB 98639.

MARTín-BRAVO \& al. (2010: 471) report this taxon from the province of León. Herein, we document additional records of this infrequently documented species in the province.

\section{Rosa stylosa Desv.}

León: Between Nogar and Saceda (Castrillo de Cabrera), 29TQG0686, $920 \mathrm{~m}$, roadside, N-NW, 09.07.2002, L. González de Paz, LEB 99523. Ca- 
brera River between Puente de Domingo Flórez and Las Vegas de Yeres (Puente de Domingo Flórez), 29TPG8198, 396 m, Rubo ulmifolii-Rosetum corymbiferae in a track border, SE, 09.05.2006, L. González de Paz, LEB 93757.

There are not any voucher specimens collected in León or housed at the Herbarium LEB. We have found an old citation from Burbia, in El Bierzo (GADOw, 1897: 394). Therefore, the present record is the second from León.

\section{Rubus castellarnaui $\mathrm{Pau}$}

León: Caprada River, between Nogar and Saceda (Castrillo de Cabrera), 29TQG0687, $870 \mathrm{~m}$, alder forest, W, 09.07.2002, L. González de Paz, LEB 99510. Silván (Benuza), 29TPG91865, 1140 m, oak forest, W, 03.07.2003, L. González de Paz, LEB 99509. Between Sigüeya and Benuza (Benuza), 29TPG9095, $1200 \mathrm{~m}$, hazel forest, NE, 28.07.2004, L. González de Paz, LEB 99508. Cabrera River in Vega de Yeres (Puente de Domingo Flórez), 29TPG8298, $400 \mathrm{~m}$, alder forest, 11.06.2005, L. González de Paz, LEB 99512. Cabrera River in Pombriego (Benuza), 29TPG8998, $470 \mathrm{~m}$, alder forest, 12.06.2005, L. González de Paz, LEB 99511. Yebra (Benuza), 29TPG9096, 729 m, hazel forest, N, 12.07.2007, L. González de Paz, LEB 99513.

This infrequent taxon has only been reported from Priaranza del Bierzo (MONASTERIO-HuELIN, 1992: 108) and Crémenes, in the Esla River valley (Alonso REDONDO, 2003: 270; CANTORAL \& al., 2011: 22).

\section{Rubus castroviejoi E. Monasterio-Huelin}

León: La Laguna, near La Baña Lake (Encinedo), 29TPG8581, $1360 \mathrm{~m}$, birch grove, NW, 04.08.2004, L. González de Paz, LEB 99505. Odollo, La Vega stream valley (Castrillo de Cabrera), 29TPG9789, $780 \mathrm{~m}$, alder forest, NE, 16.07.2005, L. González de Paz, LEB 99506. Faeda stream valley (Encinedo), 29TPG9279, 1208 m, birch forest, W-NW, 24.08.2006, L. González de Paz, LEB 99507.
We have found neither voucher specimen data nor bibliographic reports for this taxon in our province, but it has been closely collected in Zamora (near the Sanabria Lake) and Orense (Casaio, MONASTERIO-HuELIN, 1997: 151). Therefore, our voucher data provide the first record for León.

\section{Rubus gallaecicus Pau}

León: Between Losadilla and La Baña (Encinedo), 29TPG9381, $1020 \mathrm{~m}$, megaforbic community, 24.06.2004, L. González de Paz, LEB 99504.

We contribute with the third record for the province after those from Villablino to Somiedo (MoNASTERIO-Huelin, 1990: 521) and Tejeira to Peña Cabalar (GABRIEL y Galán \& Puelles, 2008: 114).

\section{Rubus henriquesii Samp.}

León: La Baña Lake (Encinedo), 29TPG8480, $1600 \mathrm{~m}$, birch forest gap, E, 06.08.2002, L. González de Paz, LEB 99498. Pathway to La Baña Lake (Encinedo), 29TPG8681, 1320 m, wayside, NW, 26.08.2003, L. González de Paz, LEB 99497. Santalavilla (Benuza), 29TPG9399, $640 \mathrm{~m}$, alder forest, 18.06.2004, L. González de Paz, LEB 99500. La Baña Lake stream (Encinedo), 29TPG8681, 1310 m, alder forest, NE, 09.07.2004, L. González de $\mathrm{Paz}$, LEB 99499. Fueyo stream, between Robledo de Losada and Nogar (Encinedo), 29TQG0384, $857 \mathrm{~m}$, Salici neotrichae-Populetum nigrae, N, 24.08.2005, L. González de Paz, LEB 99501. Valdecorrales stream, near Llamas de Cabrera (Benuza), 29TPG9497, $651 \mathrm{~m}$, alder forest, SW, 25.08.2006, L. González de Paz, LEB 99502. Espino stream, Noceda de Cabrera (Castrillo de Cabrera), 29TQG0490, $908 \mathrm{~m}$, hazel forest, S-SW, 17.07.2007, L. González de Paz, LEB 99503.

The only known references are from the Cantabrian Range: between Rioscuro and El Villar de Santiago (Monasterio-Huelin, 1990: 522); Pontón Pass (Lence PAZ, 2001: 209); Canto el Arbecho and Vega de Gustairices (both in Susañe del Sil) and Ventana Pass (DEL EGIDO \& al., 2012: 300). Our records from the southwest of León represent a significant range extension for this species. 
Rubus sampaioanus Sudre ex Samp.

León: Sotillo de Cabrera (Benuza), 29TPG8696, $949 \mathrm{~m}$, megaforbic community under walnut trees, SE, 23.05.2006, L. González de Paz, LEB 93756. Trabazos (Encinedo), 29TPG9985, 1287 m, Salix atrocinerea grove, SW, 04.09.2007, L. González de Paz, LEB 99486.

Herein, we provide the first report of this taxon in León.

\section{Rubus vagabundus Samp.}

León: Truchillas Lake River (Truchas), 29TQG0878, 1200 m, riverbank, NW, 21.07.2002, L. González de Paz, LEB 99467. Between Sigüeya and Benuza (Benuza), 29TPG9095, $1200 \mathrm{~m}$, hazel woodland, NE, 28.07.2004, L. González de Paz, LEB 99466. Between Sigüeya and Benuza (Encinedo), 29TPG9095, $1200 \mathrm{~m}$, oak forest, NE, 28.07.2004, L. González de Paz, LEB 99465. Argaño stream valley, near Santa Eulalia de Cabrera (Encinedo), 29TQG0077, $1300 \mathrm{~m}$, alder forest, N, 25.08.2004, L. González de Paz, LEB 99464. Forna (Encinedo), 29TPG9583, $1210 \mathrm{~m}$, moist grassland, SW, 11.07.2005, L. González de Paz,LEB 99468. Santalavilla (Benuza), 29TPG925992, 610 m, Smyrnium olustratum community, 16.05.2006, L. González de Paz, LEB 99469. Santa Elena chapel pathway (Benuza), 29TPG9392, $978 \mathrm{~m}$, hazel forest, NW, 11.06.2006, L. González de Paz, LEB 99470. Villarino stream, between Odollo and Llamas de Cabrera (Castrillo de Cabrera), 29TPG9693, $826 \mathrm{~m}$, hazel forest, $\mathrm{S}$, 25.08.2006, L. González de Paz, LEB 99471.

It is a species not very collected or cited in our province but it occurs frequently in the Cabrera River valley.

\section{Salvia sclarea L.}

León: Nogar (Castrillo de Cabrera), 29TQG 053864, $820 \mathrm{~m}$, slaty scree slope, E, 26.06.2005, L. González de Paz, LEB 98226.

Herein, we provide the third record from León of this taxon, which has been previously reported naturalized in Robledo de Losada (DíEz \& al., 1984: 192) and San Pedro Mallo (MARTínEZ ARIAS \&al., 2004: 271).

\section{Sedum lagascae Pau}

(= Sedum maireanum Sennen; = Sedum villosum L. subsp. aristatum (Emb. \& Maire) M.Laínz)

León: Corporales, Soña River valley (Truchas), 29TQG0887, $1240 \mathrm{~m}$, grassland with common brooms, NW, 19.06.2002, L. González de Paz, LEB 97131.

This not very frequent taxón has only been reported from Santa Colomba de Somoza and Villadangos del Páramo (Alonso Redondo \& al., 1998: 224), El Valle de las Casas (Alonso ReDONDO, 2003: 293) and Fontanos de Torío (DEL EGIDO, 2012: 329).

\section{Silene inaperta L. subsp. inaperta}

León: Between Nogar and Saceda (Castrillo de Cabrera), 29TQG0688, 906 m, Lavandulo sampaioanae-Genistetum hystricis initial facies, SW, 07.08.2007, L. González de Paz, LEB 97415.

WiLLKOMM \& LANGE (1880: 661) documented the first record for this species in León (Villafranca del Bierzo) and we contributed with the second one.

\section{Silene nocturna $\mathrm{L}$.}

León: Portela del Cuerno, near Peñarrubia (Carucedo), 29TPH811036, 860 m, Ononido pusillae-Thymetum zygidis, S-SW, 18.05.2005, L. González de Paz, LEB 97396.

It is the first report in León but nearby in Orense has been cited in Rubiana (GómEz VIGIDE, 1985: 369) and in Cornatel (GIMÉNEZ DE AZCÁRATE \& AMIGO, 1996: 136).

Sorbus latifolia (Lam.) Pers.

León: Portela del Cuerno, near Peñarrubia (Carucedo), 29TPH8103, $850 \mathrm{~m}$, border of a 
base-rich holm oak forest, 18.05.2005, L. González de Paz, LEB 98404.

It has been previously cited from northern León (ROMERo RodrígueZ, 1983: 74; PÉREZ MORALES, 1988: 91; DEL EGIDO, 2012: 342), but not in the southwestern part of the province where we have found it.

\section{Spirodela polyrrhiza (L.) Schleid.}

León: Sil River in Salas de la Ribera (Puente de Domingo Flórez), 29TPH7900, 380 m, riverbank, 11.06.2005, L. González de Paz, LEB 98153.

Cirujano \& al. (1990: 520) report this species from Carucedo. Herein, we document the second record for León.

\section{Stipa bromoides (L.) Dörfl.}

León: Salas de la Ribera (Puente de Domingo Flórez), 29TPH8102, $620 \mathrm{~m}$, base-rich holm oak forest, S, 23.06.2004, L. González de Paz, LEB 98795. Salas de la Ribera, Balouta stream valley (Puente de Domingo Flórez), 29TPH8102, 590 $\mathrm{m}$, base-rich grassland, 10.08.2009, L. GoNZÁLEZ DE PAZ, LEB 103058.

First and second records of this grass in León. It has been cited from Orense by GómEZ VIGIDE (1985: 378) and GIMÉNEZ DE AZCÁRATE \& AMIGO (1996: 140). VÁZQueZ \& DEVESA (1996: 161) do not include Orense or León in its Iberian distribution.

\section{Tolpis $x$ grosii Talavera \\ (T. barbata $\times$ T. umbellata)}

León: Sotillo de Cabrera (Benuza), 29TPG8595, 1250 m, pathway edge, SE, 11.07.2002, L. González de Paz, LEB 96283. La Vallina de Morouto, Castrillo de Cabrera, 29TQG0289, $900 \mathrm{~m}$, weed communities, W-NW, 30.07.2002, L. González de Paz, LEB 96287. Puente de Domingo Flórez (Puente de Domingo Flórez), 29TPG8098, 560 m, recently burned grassland, S-SE, 04.05.2004, L. González de Paz, LEB 96285. Puente de Domingo Flórez, 29TPG8098, $460 \mathrm{~m}$, pathway, 24.05.2005, L. González de Paz, LEB 95022. Between Sigüeya and Llamas (Benuza), 29TPG942959, $830 \mathrm{~m}$, pathway edge, 25.05.2005, L. González de Paz, LEB 95017. Villadangos, 11.06.1969, Andrés \& Carbó, LEB 4598. La Virgen del Camino, 11.06.1969, Andrés \& Carbó, LEB 4597. Onzonilla, 30TTN81, 22.10.1976, A. Penas, LEB 50040. San Martín de la Falamosa, 04.07.1977, Araceli, LEB 9169. Cueto Ancino, 30TUN05, siliceous scree, 09.08.1979, M.J. López Pacheco, LEB 16248. Cueto Ancino, 30TUN05, siliceous scree, 09.08.1979, M.J. López Pacheco, LEB 17214. Monte del Duque, 30TTM96, holm oak forest, 19.06.1983, M.E. García, LEB 19469. Torre del Bierzo, 29TQH207203, pathway edge, 30.06.2001, R. Arias, LEB 78886. Bembibre, 29TQH090233, scree, 23.06.2002, R. Arias, LEB 79235.

Whereas we have not found bibliographic records of this species from León, but the number of voucher specimens is large in LEB

\section{Trigonella gladiata Steven es M. Bieb.}

León: Salas de la Ribera (Puente de Domingo Flórez), 29TPH8002, 750 m, crack in dolomites, SW, 29.04.2004, L. González de Paz, LEB 94858. Salas de la Ribera (Puente de Domingo Flórez), 29TPH808027, 780 m, Ononido pusillae-Thymetum zygidis, S, 05.05.2005, L. González de Paz, LEB 94859.

These records extend the geographical range of this species to the southwest of León. All bibliographic records are from the Cantabrian Range: (CARBÓ \& al., 1977: 80; ROMERO RODRÍGUEZ, 1983: 77; PÉREZ MoRAlEs, 1988: 98; AlONSO REDONDO, 2003: 321).

\section{Urospermum picroides (L.) F. W. Schmidt}

León: Salas de la Ribera, El Rochouso (Puente de Domingo Flórez), 29TPH8002, 700 $\mathrm{m}$, crack in dolomites, S, 29.04.2004, L. González de Paz, LEB 96279. Salas de la Ribera (Puente de Domingo Flórez), 29TPH808027, 
$750 \mathrm{~m}$, Ononido pusillae-Thymetum zygidis, S, 05.05.2005, L. González de Paz, LEB 96277. Salas de la Ribera (Puente de Domingo Flórez), 29TPH808027, $780 \mathrm{~m}$, Ononido pusillae-Thymetum zygidis, S, 05.05.2005, L. González de Paz, LEB 96278.

DíAZ GonZÁlez \& PenAS reported the first records (1984: 151) from the Peñarrubia reservoir.

\section{Vicia amphicarpa L.}

León: La Laguna, Yeres (Puente de Domingo Flórez), 29TPH8302, 857 m, Pteridium aquilinum formations, 10.06.2006, L. González de Paz, LEB 96539.

We have only found two old bibliographic references: Villafranca del Bierzo and Astorga (GANDOGER, 1917: 104).

\section{Vicia dasycarpa Ten.}

León: Salas de la Ribera (Puente de Domingo Flórez), 29TPH8000, 380 m, weed communities, 02.05.2002, L. González de Paz, LEB 94840.

It has already been reported from León, in Selgas de Ordás reservoir, Oterico and Valencia de Don Juan (Alonso Redondo \& al., 2002: 125); Morgovejo and Prado de la Guzpeña (Alonso REDONDO, 2003: 333); and Matallana train station and Villaquilambre (DEL EGIDO, 2012: 371-372). Our report extend its range to the southwestern area of the province.

\section{ACKNOWLEDGEMENTS}

This study has been funded by Junta de Castilla y León and European Social Fund.

\section{REFERENCES}

Acedo, C. \& Llamas, F. -2006- Catálogo de plantas alóctonas en la provincia de León (NW de España) - Studia Bot. 25: 63-96.

Aedo, C. - 2003- Observaciones sobre la flora cantábrica. Unpublished, Madrid. in Anthos. 2011. Sistema de información de las plantas de España. Real Jardín Botánico, CSIC- Fundación Biodiversidad. www.anthos.es [Web. 2nd of January 2014].

Aedo, C., Aldasoro, J .J., Argüelles, J.M., Díaz Alonso, J.L., Díez Riol, A., González del Valle, J.M., Laínz, M., Moreno Moral, G., Patallo, J. \& Sánchez Pedraja, O. 1994- Contribuciones al conocimiento de la flora cantábrica, II - Fontqueria 40: 67-100.

Aedo, C., Aldasoro, J.J., Argüelles, J.M., Carlón, L., Díez Riol, A., González del Valle, J.M., Laínz, M., Moreno Moral, G., Patallo, J. \& Sánchez Pedraja, O. -2000Contribuciones al co $\neg$ nocimiento de la flora cantábrica, IV - Bol. Cien. Nat. Ridea 46: 7-119.

Aedo, C., Aldasoro, J.J., Argüelles, J.M., Carlón, L., Díez Riol, A., Gómez Casares, G., González del Valle, J.M., Laínz, M., Moreno Moral, G., Patallo, J. \& Sánchez Pedraja, O. - 2002- Contribuciones al conocimiento de la flora cantábrica, VI - Bol. Cien. Nat. Ridea 48: 775.

Alonso Redondo, R. - 2003- Valoración del estado de conservación de la vegetación y propuestas de ordenación y uso del territorio de la margen izquierda de la cuenca alta del río Esla (León) - Ser. Tesis Doc. Univ. León. Secr. Publ. Med. Aud. León. Pp. 1049.
Alonso Redondo, R., de Paz Canuria, E., Puente García, E. \& Penas, A. - 1998 - Sobre la flora de la provincia de León - Acta Bot. Malac. 23: 215-226.

Alonso Redondo, R., López Pacheco, M.J., Puente García, E. \& Penas, A. - 1999- Refe-rencias corológicas de plantas vasculares en el NW Ibérico - Acta Bot. Malac. 24: 192-195.

Alonso Redondo, R., de Paz Canuria, E., García González, M.E., Puente García, E. \& Penas, A. - 2002- Notas corológicas sobre la flora vascular de la provincia de León - Studia Bot. 21: 121-126.

Arenas Posada, J.A. \& García Martín, F. - 1993- Atlas carpológico y corológico de la subfamilia Apioideae Drude (Umbelliferae) en España peninsular y Baleares - Ruizia 12: 1-245.

Bañares, Á., Blanca, G., Güemes, J., Moreno, J.C. \& Ortiz, S. (Eds.) - 2009- Atlas y Libro Rojo de la Flora Vascular Amenazada de España. Ad. 2008 - Dir. Gen. Med. Nat. y Pol. For. Soc. Esp. Biol. Cons. Pl., Madrid. Pp. 155.

Cantó, P. - 1984- Revisión del género Serratula L. (Asteraceae) en la Península Ibérica - Lazaroa 6: 7-80.

Cantó, P. - 2012- Geobotanical synopsis of Klasea and Serratula (Asteraceae) in the Iberian Peninsula - Int. J. Geobot. Res. 2: 21-33.

Cantó, P. - 2013 - Klasea Cass. - In: Castroviejo \& al. (Eds.). Flora Iberica, vol. 20. R. Jard. Bot. CSIC, Madrid Cantoral González, A., Alonso Redondo, R. \& García González, M.E. -2011 - Datos sobre Lathraea squamaria en la provincia de León (España) - Lazaroa 32: 21-28. 
Cantoral González, A., Alonso Redondo, R. \& García González, M.E. - 2011 - Aportaciones al estudio corológico de la flora de la provincia de León - Fl. Montiber. 48: 15-20.

Carbó, R., Mayor López, M., Andrés Rodríguez, J. \& Losa Quintana, J.M. -1977- Aportaciones al catálogo florístico de la provincia de León. II - Acta Bot. Malac. 3: 63-120.

Carbó, R., Andrés, J., Llamas, F. \& Pérez, M.A. - 1987— Aportaciones al catálogo caricológico de la provincia de León - Studia Bot. 6: 68-74.

Carlón, L., Gómez Casares, G., Laínz, M., Moreno Moral, G., Sánchez Pedraja, O. \& Schne-eweiss, G.M. 2005 - Más, a propósito de algunas Orobanche L. y Phelipanche Pomel (Oroban $\neg$ chaceae) del oeste del Paleártico- Doc. Jard. Bot. Atlántico 3: 1-71.

Castroviejo, S. \& al. (Eds.) -1986-2013 - Flora Iberica. Plantas Vasculares de la Península Ibérica e Islas Baleares. - R. Jard. Bot., C.S.I.C., Madrid.

Cirujano, S., Velayos, M. \& Carrasco, M.A. - 1990Notas sobre higrófitos peninsulares, III- An. Jard. Bot. Madrid 47(2): 519-520.

Del Egido, F. - 2012 - Flora y vegetación de la cuenca del río Torío (León)[Recurso electrónico]: Cartografía y valoración de la vegetación: aplicaciones a la gestión y ordenación del territorio - Publ. Univ. León. 932 pp.

Del Egido, F., Puente García, E. \& López Pacheco, M.J. 2007 - De plantis legionensibus. Notula XXI - Lazaroa 28: $115-122$.

Del Egido, F. \& Puente, E. - 2011 - Sobre los nombres correctos de dos asociaciones homónimas: Epipactido palustris-Eriophoretum latifolii - Lazaroa 32: 181-182.

Del Egido, F., Fernández Cañedo, M., Ferreras Jiménez, N., Puente, E. \& López Pacheco, M.J. - 2012a - De Plantis legionensibus. Notula XXVII - Lagascalia 32: 298-305.

Del Egido, F., Fernández Cañedo, M., Ferreras, N., Puente, E. \& López Pacheco, M.J. - 2012b - Notas sobre flora leonesa amenazada, II - Lazaroa 33: 207-218.

Díaz González, T.E. \& Penas, A. - 1984 - De plantis legionensis. Notula I- An. Jard. Bot. Madrid 41(1): 147-154.

Gabriel y Galán, J.M. \& Puelles, M. -2008- Algunas plantas de interés de la provincia de León (España) Bot. Compl. 32: 113-116.

Gadow, H. - 1897 - In northern Spain - Adam \& Charles Black, London. Pp 421.

Gandoger, M. - 1910 - Notes sur la flore hispano-portugaise. Quatrieme voyage en Portugal - Bull. Soc. Bot. France 57: 94-100.

Gandoger, M. - 1917- Catalogue des plantes récoltées en Espagne et en Portugal pendant mes voyages de 1894 à 1912 - Paris. Pp. 378.

García Río, R. \& Navarro Andrés, F. - 1994- Flora y vegetación cormofíticas de las comarcas zamoranas del Pan, Tera y Carballeda - Studia Bot. 12: 23-202.

Giménez de Azcárate, J. \& Amigo, J. - 1996 - Inventario da flora vascular de afloramientos calíos de Galicia (Pteridophyta e Spermatophyta) - Cad. Area Ci. Biol. (Inv.). Sem. Est. Galegos XII. Ed. Do Castro, A Coruña.
Giménez de Azcárate, J., Amigo, J. \& Izco, J. — 1990Pastizales nanoterofíticos de los afloramientos calizos de Galicia: Thero-Brachypodion distachyi - Studia Bot. 9: 9-16.

Gómez Vigide, F. - 1985- Algunas aportaciones al conocimiento de la flora gallega - An. Jard. Bot. Madrid 41(2): 367-380.

Laínz, M. -1968 - Aportaciones al conocimiento de la flora gallega, VI - Publ. Inst. For. Inv. Exp. Madrid. Pp. 39.

Laínz, M. -1971 - Aportaciones al conocimiento de la flora gallega, VII - Inst. For. Inv. Exp. 12: 1-39.

Laínz, M. - 1973 - Aportaciones al conocimiento de la flora cántabro-astur, X- Bol. Inst. Est. Astur., Supl. Ci. 16: 159-206.

Laínz, M. -1974-Aportaciones al conocimiento de la flora gallega, VIII - I.N.I.A. 2: 1-26.

Lence Paz, C. - 2001 - Evaluación del estado de conservación de la vegetación del valle de Valdeburón (León). Propuestas de uso y ordenación territorial - Mem. Doc. (inéd.). Univ. León. Pp. 937.

Llamas, F. \& Acedo, C. - 1997- De plantis legionensibus. Notula XV - Studia Bot. 16: 139-141.

Llamas, F., Carbó, R. \& Andrés, J. -1984- Contribución al conocimiento de la flora de León (España) - Lazaroa 6: 283-285.

Luceño, M. - 1986- Estudios en el género Carex. I. Sección Canescentes (Fries) Christ.: C. furva Webb y C. lachenalii Schkuhr - An. Jard. Bot. Madrid 42(2): 427-440.

Martín-Bravo, S., Valcárcel, V., Vargas, P. \& Luceño, M. 2010- Geographical speciation related to Pleistocene range shifts in the western Mediterranean mountains (Reseda sect. Glaucoreseda, Resedaceae) - Taxon 59 (2): 466-482.

Martínez Arias, R., Fernández Rodríguez, A. \& García González, M.E. -2004- Nuevas citas y correcciones a Flora Iberica para plantas del NW de la provincia de León - Acta Bot. Malacitana 29: 268-273.

Mateo Sanz, G. -2006- Revisión sintética del género Hieracium L. en España, II. Sect. Sabauda - Fl. Montiber. 34: 38-49.

Mateo Sanz, G. \& del Egido, F. - 2011- Especies nuevas del género Hieracium L. (Compositae) en la provincia de León, III - Fl. Montiber. 48: 24-37.

Molina, A., Acedo, C. \& Llamas, F. -2006- Observaciones sobre el género Carex en la provincia de León (NW España) - Lagascalia 26: 25-37.

Monasterio-Huelin, E. - 1990 - Notas sobre Rubus ibéricos - An. Jard. Bot. Madrid 47(2): 521-523.

Monasterio-Huelin, E. -1992 - Fragmenta chorologica occidentalia, 4289-4293 - An. Jard. Bot. Madrid 50(1): 108.

Monasterio-Huelin, E. - 1997 - Fragmenta chorologica occidentalia, 5919-5926 - An. Jard. Bot. Madrid 55(1): 151-152.

Pérez Carro, F.J., Fernández Areces, M.P. \& Díaz González, T.E. - 1985- De plantis legionensis. Notula II - Studia Bot. 4: 137-142. 
Pérez Morales, C. - 1988 - Flora y vegetación de la cuenca alta del río Bernesga (León) - Inst. Fray Bernardino Sahagún. Excma. Dip. Prov. León, León. Pp. 437.

Romero Rodríguez, C.M. - 1983- Flora y vegetación de la cuenca alta del río Luna (León) - Min. Agric. Pesc. Alim. ICONA. Monogr. 29. Pp. 273.

Sanz Elorza, M., Dana Sánchez, E.D. \& Sobrino Vesperinas, E. (Eds.) - 2004- Atlas de las plantas alóctonas invasoras de España- Dir. Gen. Biodiversidad, Madrid. Pp. 384.

Sanz Elorza, M. \& González Bernardo, F. -2007- Contribución al conocimiento de la flora vascular alóctona de Castilla y León - Stud. Bot 26: 105-110.

Sanz Elorza, M., González Bernardo, F. \& Gavilán Iglesias, I.P. - 2008 - La flora alóctona de Castilla y León (España) - Bot. Compl. 32: 117-137.
Silva Pando, F.J. - 1994 - Flora y series de vegetación de la sierra de Ancares - Fontqueria 40: 233-388.

Talavera, S. - 1980 - El género Tolpis Adanson en Andalucía occidental - Lagascalia 9(2): 229-231.

Tutin, T.G. \& al. (Eds.) - 1968-1980,1993 - Flora Europaea, vols. 1-5 (second ed.) - Cambridge Univ. Press, London.

Vázquez, F.M. \& Devesa, J.A. - 1996- Revisión del género Stipa L. y Nassella Desv. (Poaceae) en la Península Ibérica e Islas Baleares - Acta Bot. Malacitana 21: 125-189.

Vicioso, C. -1959- Estudio monográfico sobre el género Carex en España- Bol. Inst. For. Inv. Exp. 79: 1-205

Willkomm, M. \& Lange, J. - 1880 - Prodromus florae Hispanicae, Vol. III - Schweizerbart, Stuttgart. Pp. 1144.

Received: 21 March, 2014

Accepted: 8 June 2014 